\title{
PAMA New Year's 2020: Did everything get brought to the party?
}

\author{
Toral R. Patel, $M D,^{a}$ and Jamieson $M$. Bourque, $M D, M H S^{a}$ \\ a Cardiovascular Division and the Cardiovascular Imaging Center, Department of Medicine, \\ University of Virginia Health System, Charlottesville, VA
}

Received Dec 10, 2019; accepted Dec 10, 2019

doi: $10.1007 / \mathrm{s} 12350-019-02007-0$

\section{See related article, pp. 1988-1997}

High-value healthcare requires an association of collaborators to work together to improve care, health, and costs of care by identifying, testing, and disseminating best practice care and corresponding payment models. In recent years, data have emerged identifying high healthcare costs in the USA related to an overuse of diagnostic imaging. ${ }^{1}$ With approximately $17.8 \%$ of gross domestic product spent on health care in the USA, and the second highest number of imaging scans performed, it is estimated that approximately $\$ 100$ billion per year is spent on diagnostic imaging. ${ }^{1,2}$ In response to this trend, cardiovascular societies have undertaken extensive efforts to target appropriate utilization of diagnostic imaging services, most prominently creation and refinement of Appropriateness Use Criteria (AUC). ${ }^{3,4}$ In addition to this robust response by the Cardiology community, legislation was passed over the past two years entitled: Protecting Access to Medicare Act (PAMA) of 2014, Section 218(b). This act established a new program to increase the rate of appropriate advanced diagnostic imaging services provided to Medicare beneficiaries and mandated from January 1, 2020 that providers must consult and document AUC via qualified clinical decision support mechanisms (CDSM) when ordering such modalities for Medicare Part B patients. These modalities include computed tomography (CT), positron emission tomography (PET), single-

Reprint requests: Jamieson M. Bourque, MD, MHS, Cardiovascular Division and the Cardiovascular Imaging Center, Department of Medicine, University of Virginia Health System, Box 8001581215 Lee Street, Charlottesville, VA 22908, USA; jamieson2@gmail.com J Nucl Cardiol 2021;28:2001-3.

$1071-3581 / \$ 34.00$

Copyright (C) 2019 American Society of Nuclear Cardiology. photon emission tomography (SPECT), and magnetic resonance imaging (MRI). The goal of this program is to reduce unnecessary cardiac imaging and the downstream cascade of invasive procedures, which have been shown to be costly, unsustainable, and low-yield in inappropriate circumstances.

Under PAMA, an ordering provider consults AUC prior to ordering an advanced imaging modality. Each claim requires a consultation in order for payment. Documentation of this AUC assessment is also required in order to receive reimbursement for the imaging services. Providers can access imaging AUC either by a stand-alone CDSM system or via CDSM software integrated into a practice's electronic health record (EHR). The PAMA program began with a voluntary reporting period from July, 2018 to December, 2019. Formal requirements for consultation begin on January 1, 2020, with a 1-year educational and testing period. This first year of testing requires AUC consultation; however, Centers for Medicare and Medicaid Services (CMS) will not impose penalties on furnishing providers nor use the data to determine "outliers." In the future, CMS intends to review interactions to determine "outlier' referring providers with low AUC adherence rates who may become subject to additional prior authorization processes.

In order to better assist with the transition and implementation of AUC, CDSM systems have been created to ensure an order adheres to AUC guidelines or that an exception applies. The American College of Cardiology (ACC), Society of Nuclear Medicine and Molecular Imaging (SNMMI), American Society of Nuclear Cardiology (ASNC), and American College of Radiology (ACR), who have worked diligently on fieldspecific AUC, have also outlined tools commercially available for use, including Care Select imaging, AIM Specialty health, Infinx, LogicNets, Medcurrent, Siemens Healthineers, Stanson Health, and others. Care Select Imaging has a competitive advantage as it 
includes CT and MRI and has been supported by the ACR and ACC. This product uses ACR AUC, favored in Radiology. Ideally the CDSM will integrate with the hospital EHR and utilize the AUC that most physicians or health care providers are familiar with. A significant challenge to this approach is that different groups of providers within a single health system may be familiar with a variety of AUC guidelines, and thus choosing one optimal CDSM may be impossible. For instance, the use of ACR AUC with some tools may be less familiar to many cardiologists who regularly use AUC created by the ACC. Given this, differences between societal AUC guidelines become even more important.

Moreover, with this new requirement comes significant concerns and limitations regarding EHR compatibility and integration, field-specific applicability, and quality validity. EHR and CDSM compatibility will be essential to streamline the AUC process, but this includes integration of information provided by provider documentation. Such information includes but is not limited to history of prior revascularization; electrocardiograms; baseline characteristics; and prior history of coronary artery disease (CAD). However, integration of such information will require quality indicator validation. For example, a history of CAD can be obtained from multiple resources, including, but not limited to, results from prior stress testing; coronary CTA results; coronary calcium scoring; elevated troponin levels; or a history of prior myocardial infarction or positive coronary angiography. Given these limitations and relatively few studies focusing on the use of AUC in quality improvement projects, future research is needed that assesses appropriateness and AUC adherence data to inform quality goals, standardize care pathways, evaluate ordering context (inpatient vs outpatient, community vs academic), and model the financial implications of moving the burden of reducing inappropriate use from the responsibility of payers to providers. Data can also inform if reducing rarely appropriate testing increases or maximizes utilization of appropriate medical therapy.

In this context, Divakaran et $\mathrm{al}^{5}$ sought to address some of the issues posed by the upcoming PAMA legislation. In their single-center study of 288 subjects, they determined appropriateness of advanced cardiovascular imaging tests ordered in the inpatient clinical setting and evaluated whether a CDSM enhanced this determination. The authors utilized a self-developed pilot CDSM system and assessed the effectives of the EHR alone compared to the EHR plus CDSM. They found that the EHR alone was insufficient to deem appropriateness in $12.8 \%$ of studies using the 2013 Multimodality AUC. ${ }^{4}$ The most common reason that appropriateness could not be determined was inability to determine exercise tolerance $(59.5 \%)$. In contrast, the CDSM system alone was sufficient in only $14.2 \%$ of cases; the most common reason for failure was that the exam indication was not addressed by the AUC criterion. Of note, only $1 \%$ of the studies ordered were deemed rarely appropriate. The study by Divakaran et al adds to the existing literature assessing current ordering practices and how a CDSM enhances the workflow in deeming a diagnostic test as appropriate. The most common reason appropriateness could not be determined after review with the EHR and the pilot CDS (4.2\% of the cases) was that there was no AUC indication published that matched the clinical reason for the exam $(83.3 \%){ }^{5}$ Such clinically appropriate cases included pre-operative testing with a coronary CTA prior to cardiac and non-cardiac surgery and evaluation of spontaneous coronary artery disease (SCAD); these are addressed in the updated ACC 2018 AUC criterion. ${ }^{3}$

This analysis also highlights the generalizability challenges in applying this standard to all populations. As an example, the physician reviewers agreed on $62.5 \%$ of cases without known CAD compared with $75.7 \%$ of cases with known CAD $(P=.021) .^{5}$ This study had a higher than average percentage with known CAD (52.8\%), likely due to the inpatient venue. The performance at an academic tertiary center also potentially leads to greater heterogeneity in types of studies ordered and high proportion of physicians-in-training ordering the studies. Conducting the study in an inpatient tertiary care center may limit generalizability of these findings to outpatient/ambulatory, same-day discharge, and primary/secondary centers. Given the vast differences in specialty and procedural expertise and care pathways, future research on diagnostic imaging trends by provider would be crucial in identifying inappropriate studies ordered. Rozanski et al showed in 2013 that there is a marked progressive temporal decline in the prevalence of abnormal stress SPECT-MPI studies, ranging from $40.9 \%$ in 1991 to only $8.7 \%$ in 2009 $(P<.0001)$, and a parallel decline in the prevalence of ischemic SPECT-MPI studies, ranging from $29.6 \%$ in 1991 to only $5.0 \%$ in $2009(P<.0001)$ in both the outpatient and inpatient populations. ${ }^{6}$ Likewise, the PROMISE trial also displayed that majority of patients undergoing testing with known CAD flows out of the inpatient status, which is conversely different from lowrisk low prevalence of disease in the outpatient setting. ${ }^{7,8}$ Therefore, further evaluation of which diagnostic modality is most appropriate in low-risk vs high-risk patient populations with and without known CAD is important.

In addition to diversity in environment, there was a substantial variation in inter-observer agreement regarding appropriateness of the study ordered. This finding highlights the challenge in applying these 
standards across all cardiologists and non-cardiologists ordering these tests. In order to minimize disagreement, education programs and clear EHR documentation recommendations will be necessary, including specific characteristics about presenting ischemic symptoms, CAD history, and previous ischemic testing. Interestingly, only $1 \%$ of the studies ordered were deemed rarely appropriate. This low rate of lower-value studies supports prior work by Kini et al who noted a significant decline in overall and low-value diagnostic cardiovascular testing in a sample of Medicare fee-for-service beneficiaries. ${ }^{9}$ These data raise the question of whether this legislation and mandated timeline are really necessary.

Although the current study demonstrates gaps that future and current CDSM systems may need to fill in order to properly assess appropriateness, there are a few concerns. For instance, the CDSM used in this study is not sufficiently described to ascertain how it differs from commercially available products. It would be beneficial to utilize an approved CDSM in a similar study and determine if the findings are consistent. This would also provide an opportunity to validate quality metrics and address the challenges posed in integrating the system. CDSM systems that utilize a multimodality approach and integrate both ACR and ACC AUC criteria in regards to PET, SPECT, CT, and MRI modalities will have a competitive advantage compared with those systems which are reflective of only one or two modalities and utilize a single society's AUC.

Ongoing studies of AUC and CDSM systems are much needed to better understand and close the gaps and address the challenges we are facing on the road to fulfilling the PAMA mandate. Utilizing CDSM systems will reshape the ambulatory strategy, affect documentation, redefine the approach to utilize guidelines and AUC, and require re-evaluation of the need for repeat studies and further testing if the initial test is deemed appropriate. With a variety of AUC criterion present, are there truly a significant number of inappropriate studies ordered? As the data comes in to light, so will the necessity of educational programs geared toward providers to help them understand AUC and CDSM models and document suitably in the EHR. Despite these concerns, the road to PAMA implementation is fast approaching, whether it is needed or not and whether we are ready or remain unprepared.

\section{References}

1. Papanicolas I, Woskie LR, Jha AK. Health care spending in the United States and other high-income countries. JAMA 2018;319:1024-39.

2. Braga L, Vinci B, Leo CG, Picano E. The true cost of cardiovascular imaging: Focusing on downstream, indirect, and environmental costs. Cardiovasc Ultrasound 2013;11:10.

3. Hendel RC, Lindsay BD, Allen JM, Brindis RG, Patel MR, White L, et al. ACC appropriate use criteria methodology: 2018 update. J Am Coll Cardiol 2018;71:935-78.

4. Hendel RC, Patel MR, Allen JM, Min JK, Shaw LJ, Wolk MJ, et al. Appropriate use of cardiovascular technology: 2013 ACCF appropriate use criteria methodology update: A report of the American College of Cardiology Foundation appropriate use criteria task force. J Am Coll Cardiol 2013;26:1305-17.

5. Divakaran S, Singh A, DeFilippis E, Churchill T, Cuddy S, Ge Y, et al. Appropriateness of inpatient stress testing: Implications for development of clinical decision support mechanisms and future criteria. J Nucl Cardiol 2019. https://doi.org/10.1007/s12350-01901955-x.

6. Rozanski A, Gransar H, Hayes SQ, Min J, Friedman JD, Thomson LE, et al. Temporal trends in the frequency of inducible myocardial ischemia during cardiac stress testing: 1991 to 2009. J Am Coll Cardiol 2013;61:1054-65.

7. Douglas PS, Hoffman U, Patel MR, Mark DB, Al-Khalidi HR, Cavanaugh B, et al. Outcomes of anatominal versus functional testing for coronary artery disease. N Engl J Med 2015;372:1291300 .

8. Hoffmann U, Ferencik M, Udelson JE, Picard MH, Truong QA, Patel MR, et al. Prognostic value of noninvasive cardiovascular testing in patients with stable chest pain: Insights from the promise trail. Circulation 2017;135:2320-32.

9. Kini V, Viragh T, Magid D, Masoudi FA, Moghtaderi A, Black B, et al. Trends in high- and low-value cardiovascular diagnostic testing in fee-for-service medicare, 2000-2016. JAMA. 2019;2:e1913070.

Publisher's Note Springer Nature remains neutral with regard to jurisdictional claims in published maps and institutional affiliations. 